\title{
Local Vaporization at the Cut Front at High Laser Cutting Speeds
}

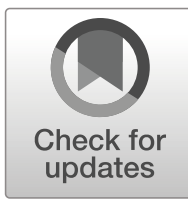

\author{
Oliver Bocksrocker 1,2,3 (D) Peter Berger ${ }^{2} \cdot$ Steffen Kessler $^{3} \cdot$ Tim Hesse $^{3}$. \\ Volker Rominger ${ }^{3}$. Thomas Graf ${ }^{2}$
}

Accepted: 3 March 2020 / Published online: 12 March 2020

(C) The Author(s) 2020

\begin{abstract}
High-speed videos of the cut front and spectrometric measurements were applied to detect local vaporization on the cut front at high cutting speeds to show that with increasing feed rate, temporally short and intense flashes are generated by vaporization phenomena on the upper part of the cut front. The latter are accompanied by the emergence of an interrupted striation pattern on the surface of the cutting edge. The findings support the assumption that local vaporization at the cut front might be the cause for reduced quality of the cutting process at elevated cutting speeds. The observation of vaporization serves as a diagnostic method to anticipate a fail cut and the interrupted striation pattern.
\end{abstract}

Keywords Laser cutting · Vaporization - Camera observation · Spectral emission · Interrupted striations

\section{Introduction}

In the area of laser cutting, the structure of the striation pattern is one of the most important quality factors of the cutting edge. At the fusion cutting process with a solidstate laser, striations can be interrupted at high cutting speeds in thick metal sheets [1]. Previous investigations on the influence of the geometry of the laser cut front on the absorbed intensity and the gas flow suggest that the change of the characteristic

Oliver Bocksrocker

oliver.bocksrocker@trumpf.com

1 Graduate School of Excellence advanced Manufacturing Engineering (GSaME), University of Stuttgart, Nobelstr. 12, 70569 Stuttgart, Germany

2 Institut für Strahlwerkzeuge (IFSW), University of Stuttgart, Pfaffenwaldring 43, 70569 Stuttgart, Germany

3 TRUMPF Werkzeugmaschinen GmbH + Co.KG, Johann-Maus-Straße 2, 71252 Ditzingen, Germany 
striation pattern is most probably induced by the changed energy deposition and not by the geometry-dependent gas dynamics [2].

In a regular fusion cutting process of steel, striations are generated that continuously extend from the top to the bottom of the cutting edge, as shown in Fig. 1-a. However, with increasing cutting velocity, the striations develop an interrupted pattern, as shown in Fig. 1-b. The so-called interrupted striations do not run from the top to the bottom of the cutting edge continuously. This constitutes a major deficit of the quality of the cutting edge [1].

The transformation of the striation pattern in the example illustrated in Fig. 1 a) and b) was generated by increasing the velocity from $v_{\text {low }}=9.5 \mathrm{~m} / \mathrm{min}$ to $\mathrm{v}_{\text {medium }}=11 \mathrm{~m} /$ $\min$. At the further increased cutting velocity $v_{\text {high }}=12.5 \mathrm{~m} / \mathrm{min}$ the interrupted striation pattern is even more pronounced (Fig. $1 \mathrm{c}$ )), and the interruption is closer to the top surface of the metal sheet. All other parameters were kept constant, see Table 1. Note that the characteristic pattern of interrupted striations is only known from the cutting process with a solid-state laser [3, 4].

There are different approaches to explain the origin of the interrupted striation pattern, all of which mention the influence of vaporization on the process behavior:

Olsen et al. [4, 5] suggest that a keyhole is formed in the lower part of the cut front at high cutting rates and high intensities. The assumption is supported by Petring et al. [6, 7] who estimate the surface temperature of the cut front to be highest near the bottom. This is in strong agreement with the experiments of Onuseit et al. [8, 9] who measured line emissions of iron emitted from the surface of the cut front for the $\mathrm{CO}_{2}$ laser cutting process. With increasing cutting velocity, they showed an increase of temperature up to boiling temperature of iron and that the temperature is highest near the bottom of the cut front.

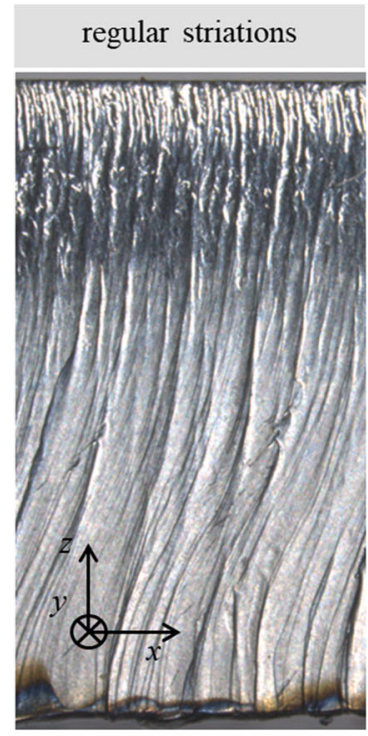

a) $v_{\text {low }}$

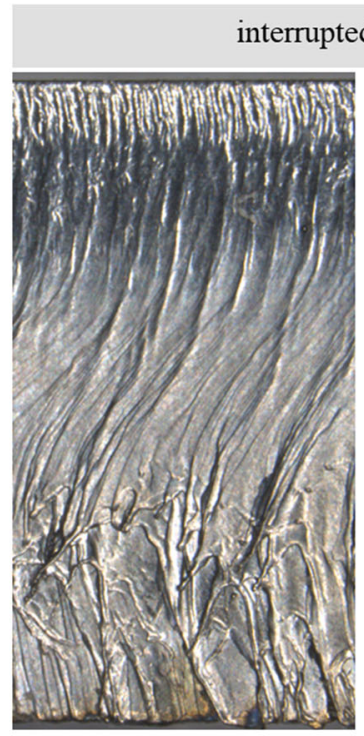

b) $v_{\text {medium }}$

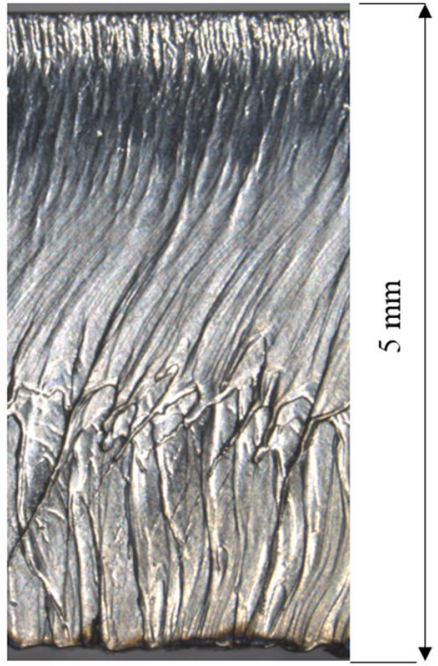

c) $v_{\text {high }}$

Fig. 1 Regular striations on the smooth surface of the cutting edge at a cutting velocity of $v_{\text {low }}$ (a) and interrupted striations at $v_{\text {medium }}(\mathrm{b})$ and at $v_{\text {high }}(\mathrm{c})$. Parameters: see Table 1 
Table 1 Cutting parameters

\begin{tabular}{lll}
\hline parameter & value & unit \\
\hline material & EN 1.0038 & - \\
sheet thickness & 5 & $\mathrm{~mm}$ \\
laser type & TruDisk 8001 & - \\
beam parameter product & 4 & $\mathrm{~mm} * \mathrm{mrad}$ \\
laser power & 8000 & $\mathrm{~W}$ \\
focal length & 200 & $\mathrm{~mm}$ \\
focal position & -1.3 & $\mathrm{~mm}$ \\
focus diameter & 210 & $\mu \mathrm{m}$ \\
nozzle diameter & $2.7(\mathrm{conical})$ & $\mathrm{mm}$ \\
distance from nozzle to work piece & 0.7 & $\mathrm{~mm}$ \\
cutting gas & $\mathrm{N}_{2}$ & - \\
pressure of cutting gas & 16 & $\mathrm{bar}$ \\
cutting velocity $v_{\text {low }}$ & 9.5 & $\mathrm{~m} / \mathrm{min}$ \\
cutting velocity $v_{\text {medium }}$ & 11 & $\mathrm{~m} / \mathrm{min}$ \\
cutting velocity $v_{\text {high }}$ & 12.5 & $\mathrm{~m} / \mathrm{min}$ \\
\hline
\end{tabular}

In contrast, Sparkes et al. [10] assume that a temporary keyhole inside the cut kerf is formed in the upper part of the cut front as cutting speeds increase and molten material accumulates inside the cut kerf. The flow of the molten material around the keyhole is suspected to be responsible for the interrupted striation pattern.

As shown in the camera observations reported in [11] for cutting with a $\mathrm{CO}_{2}$ laser, short and intense process emissions from the cut front, so-called flashes, indicate plasma events in the upper part of the cut front. Local vaporization accompanied by short and intense flashes was also found on the top surface of the humps on the front wall of the keyhole for laser welding by Kaplan et al. [12, 13].

The vaporization associated with these flashes so far, however, was neither referred to as the cause of an interrupted striation pattern while cutting with a solid-state laser nor it was differentiated whether the flashes are caused by the heating or ionization of the cutting gas or by hot vaporized metal.

Here we therefore report on investigations to identify whether and where vaporization takes place at the cutting front at high cutting speeds. To do this, flashes on the cut front were localized by analyzing high-speed videos at different cutting speeds. In addition, spectrometric measurements were used to determine the temperature and to attribute line emissions to the hot vapor of iron. It is shown that vaporization of metal is locally concentrated in the upper part of the cut front at high cutting speeds. The applied diagnostics can also be used to anticipate the formation of interrupted striations and therefore prevent fail cuts by reducing the cutting speed as soon as flashes are detected.

\section{Methodology}

To answer the question of whether interrupted striations coincide with local vaporization on the cut front at high cutting speeds, process emissions were first localized by 
high-speed imaging. The temperature inside these detected bright zones was then determined by means of spectrometric measurements.

\section{Localization of Flashes on the Cut Front by High-Speed Imaging}

Hot vapor of iron $(\mathrm{Fe} I)$ is known to emit characteristic lines in the spectral range between $500 \mathrm{~nm}$ and $550 \mathrm{~nm}[8,9]$. The camera used to image the cutting front was therefore equipped with a bandpass filter transmitting in the range between $500 \mathrm{~nm}$ and $550 \mathrm{~nm}$. The experimental setup with the imaging camera used to observe the cut front is sketched in Fig. 2, details are described in [14]. The $x$-z plane is identical to the plane of symmetry in the cut kerf, which is spanned by the axis of the laser beam and the moving direction of the work piece. The camera is orientated at a tilt of $15^{\circ}$ with respect to the top surface of the metal sheet.

Before the main cut was performed from which the high-speed video was taken, the metal sheet was first cut for only $1 \mathrm{~mm}$ from the edge of the sheet to generate a solidified cut front. The camera was then focused on the solidified cut front. The position of the laser head and the camera were kept constant, while the machine table with the clamped metal sheet was moved in negative $x$ direction for cutting. By this, the position of the cut front was kept constant with respect to the position of the camera and ensured well focused images. The cutting parameters and the parameters for the camera observation are listed in Table 1 and Table 2, respectively.

To compare a process exhibiting regular striations to processes leading to interrupted striations on the surface of the cutting edge, all parameters were kept constant except for the cutting velocity, which was increased from $v_{\text {low }}$ to $v_{\text {medium }}$ and $v_{\text {high }}$ (see values in Table 1).

In order to localize the area of maximum process emissions on the cut front, the gray values of each pixel were compared over one hundred frames and the maximum value

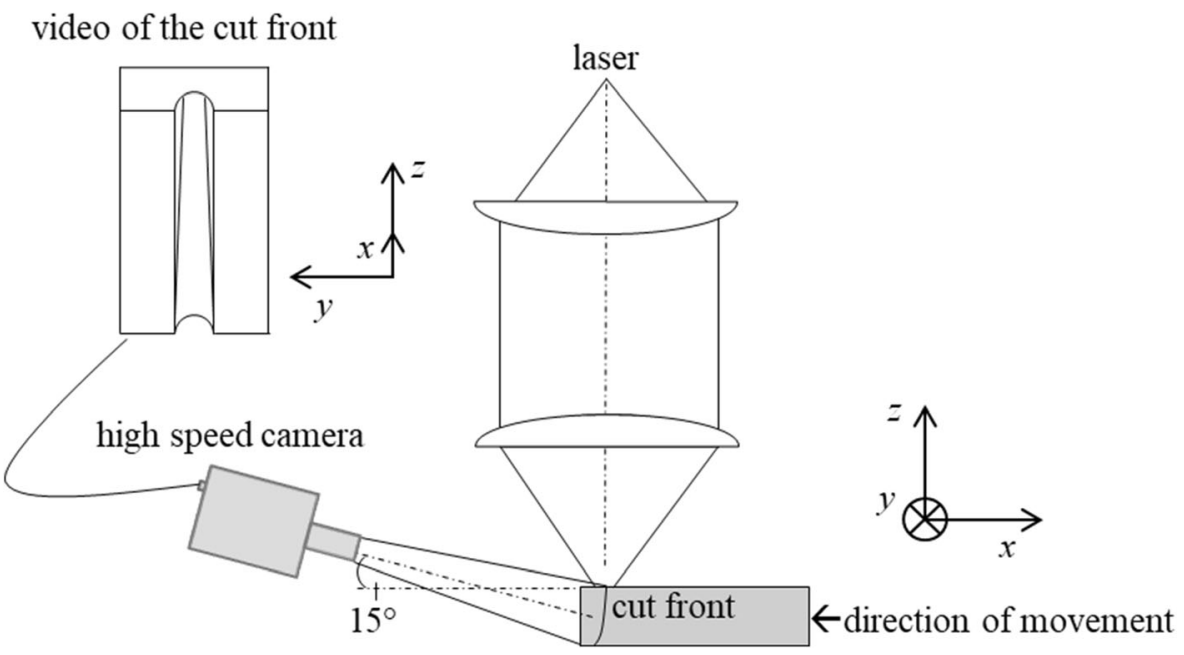

Fig. 2 High-speed imaging setup for the observation of the cut front in the plane of symmetry ( $x-z$ plane). During the experiments, the metal sheet was moved in $-\mathrm{x}$ direction whereas camera and the laser head were stationary 
Table 2 Observation parameters for the high-speed camera

\begin{tabular}{lll}
\hline parameter & value & unit \\
\hline sensor type & CMOS & - \\
frame rate & 10 & $\mathrm{kHz}$ \\
exposure time & 1 & $\mu \mathrm{s}$ \\
optical resolution & 12.2 & $\mu \mathrm{m} / \mathrm{pixel}$ \\
bandpassfilter & $525 \pm 25$ & $\mathrm{~nm}$ \\
\hline
\end{tabular}

was adopted for the given pixel. To smoothen the signal the gray values of each pixel was first averaged over three consecutive frames by a moving average.

The area exhibiting the highest process emissions on the cut front is referred to as the region of interest (ROI) in the following.

\section{Spectral Analysis of Process Emissions}

The temperature at the location of the flashes in the ROI on the cut front, was determined by two different spectroscopic approaches. On the one hand, the temperature was determined by a gray body fit to the thermal emission out of the ROI. On the other hand, the intensity of the emission lines was compared to determine the temperature of the iron vapor. The setup used for these experiments is shown in Fig. 3. The focusing optics for the spectral analysis was again orientated at a $15^{\circ}$ tilt with respect to the surface of the sheet, to observe the cut front from the same direction as with the high-speed camera used before. The optical axis of the spectrometer was again in the plane of symmetry of the cut kerf. And again, the laser head and the measurement optics remained fixed at one position while the metal sheet was moved in negative $\mathrm{x}$ direction (see Fig. 3).

Considering the $15^{\circ}$ tilt of the camera with respect to the surface of the work piece and the assumption that the angle between the cut front and the surface was between $70^{\circ}$ and $90^{\circ}$, the observation angle was estimated to range between $0^{\circ}$ and $20^{\circ}$ with respect to the surface normal of the cut front. Under these conditions, the emission coefficient $\varepsilon$ can be approximated by the one at normal incidence [8].

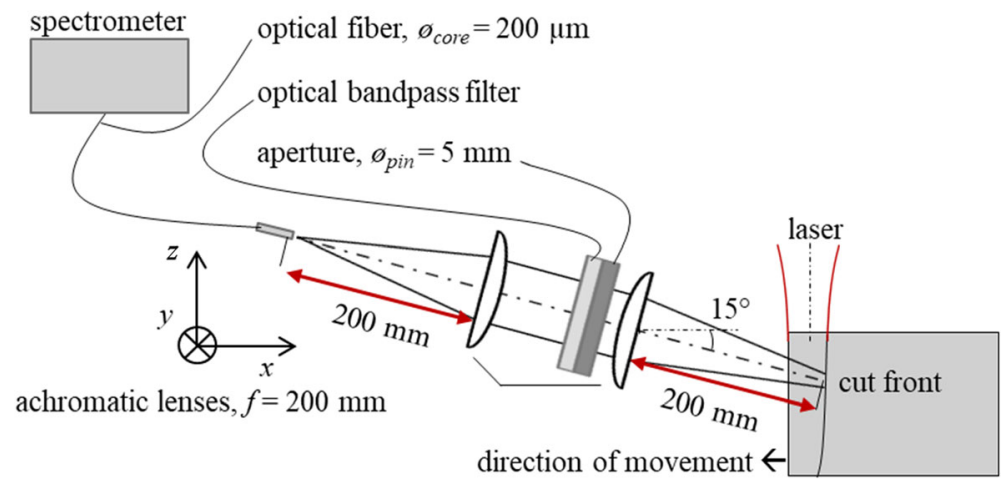

Fig. 3 Experimental setup for the spectral analysis of the process emissions from the cut front 
The parameters used for the spectrometric measurements are listed in Table 3. The sensor of the spectrometer was protected by reducing the amount of collected light with the bandpass filter specified below and an aperture with a diameter of $5 \mathrm{~mm}$. The achromatic lenses, each with a focal length of $200 \mathrm{~mm}$, were positioned at $200 \mathrm{~mm}$ from the optical fiber and $200 \mathrm{~mm}$ from the cut front. The magnification therefore was $1: 1$. The diameter of the measuring spot on the cut front was $200 \mu \mathrm{m}$ and was adjusted at a position at $1.9 \mathrm{~mm}$ below the upper surface of the metal sheet. The spectral measurements were averaged each time over ten frames.

In order to detect the emission lines of the stimulated iron vapor on top of the thermal radiation emitted from the interaction zone, the range for the spectral analysis was chosen to be $380 \mathrm{~nm}-730 \mathrm{~nm}$. This spectral range includes emission lines of iron $[8,9]$ and lies within the sensitivity range of the charge-coupled device (CCD) sensor. For all measurements the raw signal of each pixel was at least $10 \%$ of the maximum gray value (255 counts at 8 bit).

The temperature of the cut front in the ROI was estimated by means of spectroscopy.

To this end, the spectral radiance

$$
L_{p r}(\lambda)=K(\lambda) \cdot\left(I_{p r}\left(\lambda, T_{p r}\right)-I_{d a}(\lambda)\right)
$$

of the process at the temperature $T_{p r}$ must be derived from the measured raw spectrum $I_{p r}\left(\lambda, \mathrm{T}_{\mathrm{pr}}\right)$ emitted by the process. Here the dark spectrum $I_{d a}(\lambda)$ corresponds to the response of the spectrometer without illumination. The raw signal of the sensor was calibrated using a tungsten strip lamp. The calibration function

$$
K\left(\lambda, T_{r a}\right)=\frac{L_{t s}\left(\lambda, T_{r a}\right)}{I_{r e f}(\lambda)-I_{d a}(\lambda)}
$$

relates the measured reference raw spectrum $I_{r e f}(\lambda)$ of the tungsten strip lamp and the dark spectrum $I_{d a}(\lambda)$ to the known spectral radiance $L_{t s}(\lambda$,) of the tungsten lamp at its known radiance temperature of $T_{r a}=2500 \mathrm{~K}$. In order to calibrate the measurement setup to the absolute temperature of the tungsten strip inside the lamp one further needs

Table 3 Observation parameters for the spectrometer

\begin{tabular}{lll}
\hline parameter & value & unit \\
\hline spectral range & $380-730$ & $\mathrm{~nm}$ \\
sensor type & $\mathrm{CCD}$ & - \\
record frequency & 500 & $\mathrm{~Hz}$ \\
exposure time & 1 & $\mathrm{~ms}$ \\
optical resolution & 0.3 & $\mathrm{~nm} / \mathrm{pixel}$ \\
bandpass filter (FWHM) & $507.5 \pm 187.5$ & $\mathrm{~nm}$ \\
aperture & 5 & $\mathrm{~mm}$ \\
focal length, both lenses & 200 & $\mathrm{~mm}$ \\
fiber diameter & 200 & $\mu \mathrm{m}$ \\
magnitude & $1: 1$ & - \\
\hline
\end{tabular}


to take into account the emissivity $\varepsilon_{t}(\lambda)$ of the tungsten strip and the spectral transmission $\tau_{b}(\lambda)$ of the glass bulb of the lamp according to

$$
\left.L_{t s}\left(\lambda, T_{r a}\right)=\varepsilon_{t}(\lambda) \cdot \tau_{b}(\lambda)\right) \cdot L_{b l}\left(\lambda, T_{t s}\right)
$$

The transmission $\tau_{b}(\lambda)$ of the glass bulb of the lamp was derived from [15]. The emissivity of the tungsten $\varepsilon_{t}(\lambda)$ was derived from [16]. The spectral radiance of a black body is given by

$$
L_{b l}\left(\lambda, T_{t s}\right)=\frac{2 \cdot c^{2} \cdot h}{\lambda^{5} \cdot\left(e^{\frac{h \cdot c}{\lambda \cdot k \cdot T_{t s}}}-1\right)}
$$

where $c$ is the speed of light, $h$ is Planck's constant, $k$ is Boltzmann's constant and $\lambda$ the wavelength of the radiation [17]. All the constants are given in Table 4. The temperature on the surface of the tungsten strip was derived from eqs. (3) and (4) at the wavelength $\lambda=650 \mathrm{~nm}$, at which the radiance temperature $T_{r a}=2500 \mathrm{~K}$ is known, and was found to be $T_{t s}=2790 \pm 15 \mathrm{~K}$.

The error estimation of $T_{t s}=2790 \pm 15 \mathrm{~K}$ results from the minimum and the maximum values of the uncertainty of the radiance temperature $\Delta T_{r a}= \pm 8 \mathrm{~K}$ and the uncertainty of the transmissivity of the glass bulb $\Delta \tau_{b}= \pm 0.0005$, both are taken from the data sheet of the manufacturer [15]. The uncertainty of the emissivity of tungsten $\Delta \varepsilon_{T}= \pm 0.0082$ was extrapolated from a graph in [16].

Knowing $T_{t s}$, the raw spectrum $I_{p r}$ can be calibrated with eq. (1) and (2) to obtain the spectral radiance $L_{\lambda, p r}$ Under the assumption of a temporally and spatially constant process temperature $T_{p r}$ on the surface of the cut front in the ROI during the measurement, the spectral radiance

$$
L_{p r}\left(\lambda, T_{p r}\right)=K\left(\lambda, T_{r a}\right) \cdot\left(I_{p r}\left(\lambda, T_{p r}\right)-I_{d a}(\lambda)\right)^{!}=\varepsilon_{p r} \cdot L_{b l}\left(\lambda, T_{p r}\right)
$$

is described by a gray body, where the emissivity of the process $\varepsilon_{p r}$ was assumed to be constant as well.

Table 4 Specific values to determine the temperature of the process $T_{p r}$.

\begin{tabular}{lll}
\hline parameter & value & unit \\
\hline radiance temperature of the tungsten strip lamp $T_{r a}$ and its uncertainty $\Delta T_{r a}[15]$ & $2500 \pm 8$ & $\mathrm{~K}$ \\
temperature on the surface of the tungsten strip $T_{t s}$ & $2790 \pm 15$ & $\mathrm{~K}$ \\
speed of light $c_{0}$ & $2.99 \times 10^{8}$ & $\mathrm{~m} / \mathrm{s}$ \\
Planck's constant $h$ & $6.626 \times 10^{-34}$ & $\mathrm{~J}{ }^{*} \mathrm{~s}$ \\
Boltzmann's constant $k$ & $1.38 \times 10^{-23}$ & $\mathrm{~J} / \mathrm{K}$ \\
emissivity of tungsten $\varepsilon_{T}$ and its uncertainty $\Delta \varepsilon_{T}$ at a wavelength of $650 \mathrm{~nm}$ & 0.4278 & - \\
$\quad$ and a temperature of $2800 \mathrm{~K}[16]$ & \pm 0.0082 & - \\
transmissivity of the bulb of fused silica $\tau_{b}$ and its uncertainty $\Delta \tau_{b}$ at a & 0.93 & \pm 0.0005 \\
$\quad$ wavelength of $650 \mathrm{~nm}[15]$ & & \\
\hline
\end{tabular}


In the experiments, the temperature of the process $T_{p r}$ and the emissivity of the process $\varepsilon_{p r}$ were both determined by a least-square fit of eq. (5) to the measured spectra.

The determination of the temperature was performed to check whether the temperature on the surface of the cut front reaches boiling temperature when local flashes appear. The detection of local flashes is discussed in the following section.

\section{Results}

In order to determine the location of the flash phenomena at the cut front, high-speed videos of the process were recorded. The area was then examined for vaporization.

\section{Local Flashes on the Cut Front}

Sequences of single frames (1) to (5) taken from the high-speed recording of the cut front with cutting speeds of $v_{\text {low }}, v_{\text {medium }}$ and $v_{\text {high }}$ are shown in Fig. 4, Fig. 5 and Fig. 6, respectively.

It can be seen in Fig. 4, that at a cutting velocity of $v_{\text {low }}$ only a few and weakly visible process emissions occur in the upper part of the cut front. In contrast, temporally short phenomena of high brightness, referred to as flashes, can be seen on the cut front at $v_{\text {medium }}$ in Fig. 5 and at $v_{\text {high }}$ in Fig. 6 . For both cutting velocities $v_{\text {medium }}$ and $v_{\text {high }}$, the

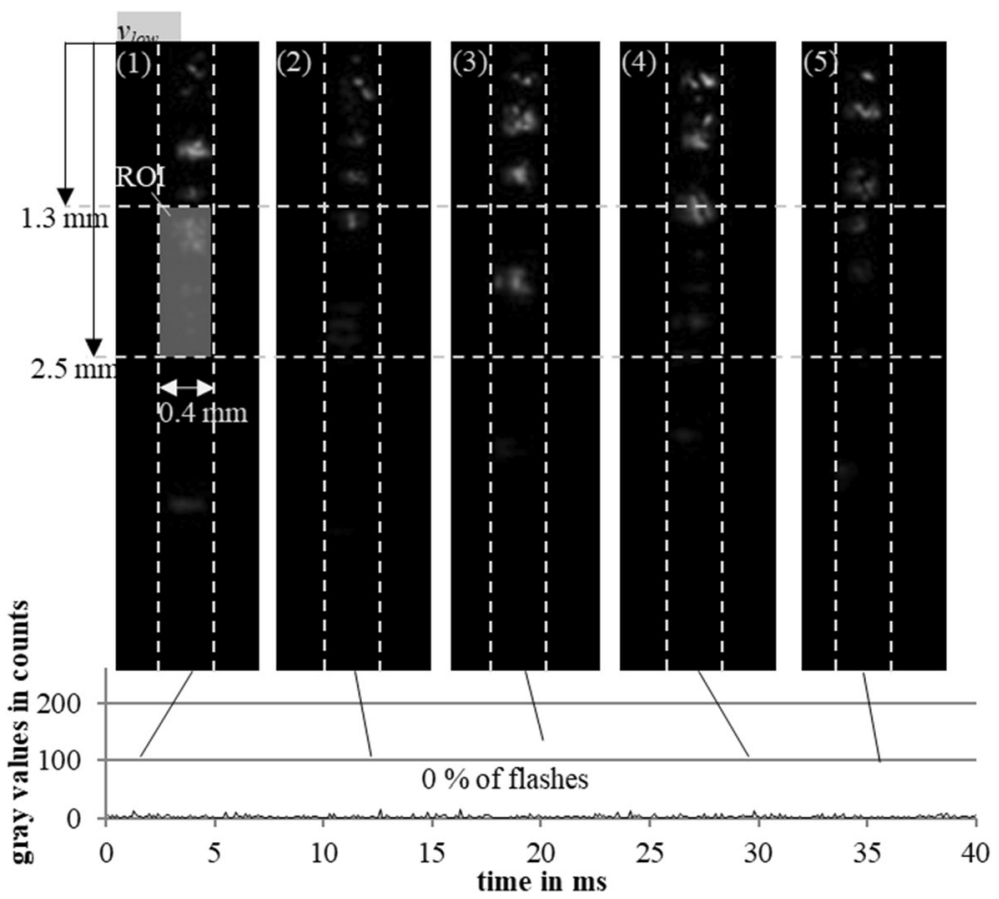

Fig. 4 Sequences of single frames taken from high-speed videos showing the cut front at the cutting velocity $v_{\text {low }}$ The vertical dashed lines indicate the edges of the cut kerf. The horizontal dashed lines show the upper and lower limits of the ROI. The diagrams below the images show the spatial average of the gray values of all pixels in the ROI of each frame plotted over time 


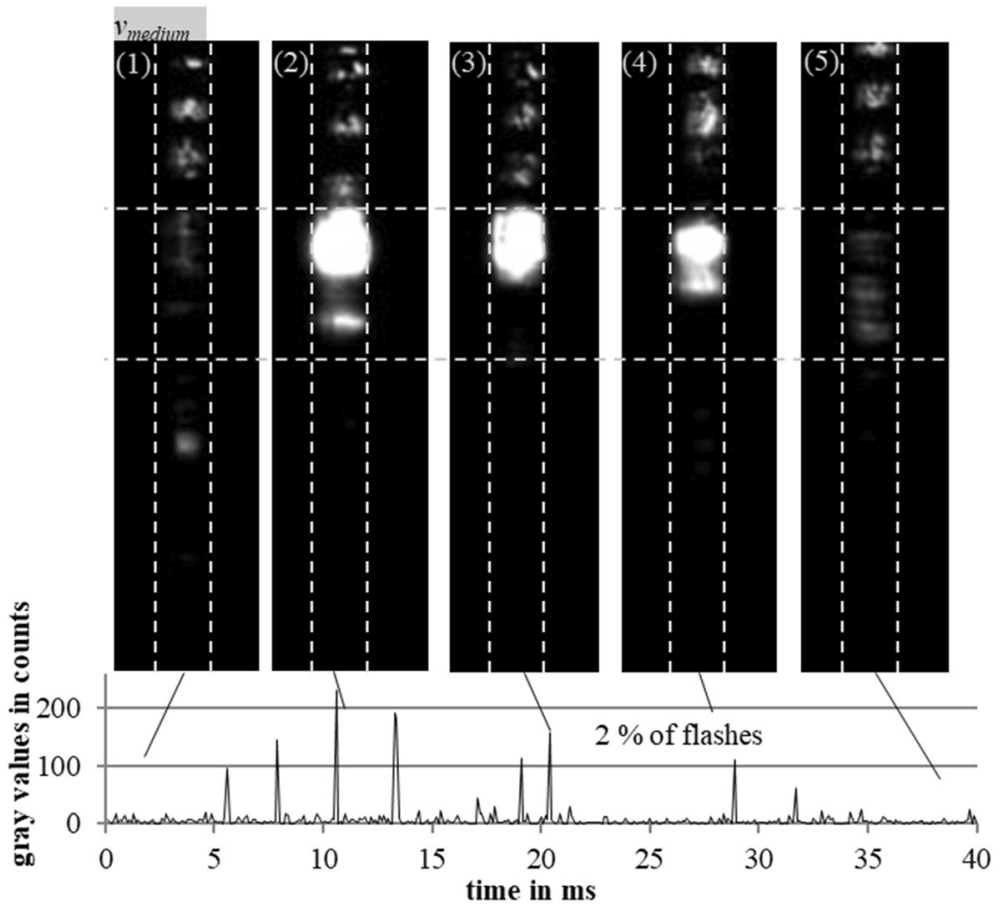

Fig. 5 Sequences of single frames taken from high-speed videos showing flashes on the cut front at the cutting velocity $v_{\text {medium }}$. The vertical dashed lines indicate the edges of the cut kerf. The horizontal dashed lines show the upper and lower limits of the ROI. The diagrams below the images show the spatial average of the gray values of all pixels in the ROI of each frame plotted over time

visible process emissions are concentrated in the upper part of the cut front. This region is considered as the region of interest (ROI) and is located between $-1.3 \mathrm{~mm}$ and $2.5 \mathrm{~mm}$ below the top surface of the metal sheet. The diagrams located below the pictures in Figs. 4, 5 and 6 show the temporal evolution of the mean gray scale values as given by the spatial average over all pixels in the ROI. The ROI is between $-1.3 \mathrm{~mm}$ and $-2.5 \mathrm{~mm}$ below the top edge of the sheet in the $\mathrm{z}$ direction and covers $24 \%$ of the surface of the cut front.

Mean gray scale values exceeding 100 (39\% of the peak value 255) in the ROI are referred to as flashes and can be counted in $2 \%$ of the images of the video at $v_{\text {medium }}$. The intensity and quantity of these flashes even increase for $v_{\text {high }}$, as shown in Fig. 6. For this cutting speed $4 \%$ of all images exhibited flashes that exceed a mean gray scale value of 100 .

The maximum duration of each of the flashes in the videos was between one and three frames, making a total of less than 0.3 milliseconds per flash. It is possible that the duration of the flashes is much shorter, which cannot be determined due to the limitation of the frame rate of the high-speed camera. Nevertheless, the occurrence of flashes at high cutting speeds can reasonably be observed with this method.

Figure 7 shows so called "stacked" images of the process emissions on the cut fronts at the cutting velocities of $v_{\text {low }}$ (a), $v_{\text {medium }}$ (c) and $v_{\text {high }}$ (e). These images are generated by comparing the gray values of each pixel over one hundred frames and selecting the maximum value of the pixels to form the stacked image. In addition, the images of the 


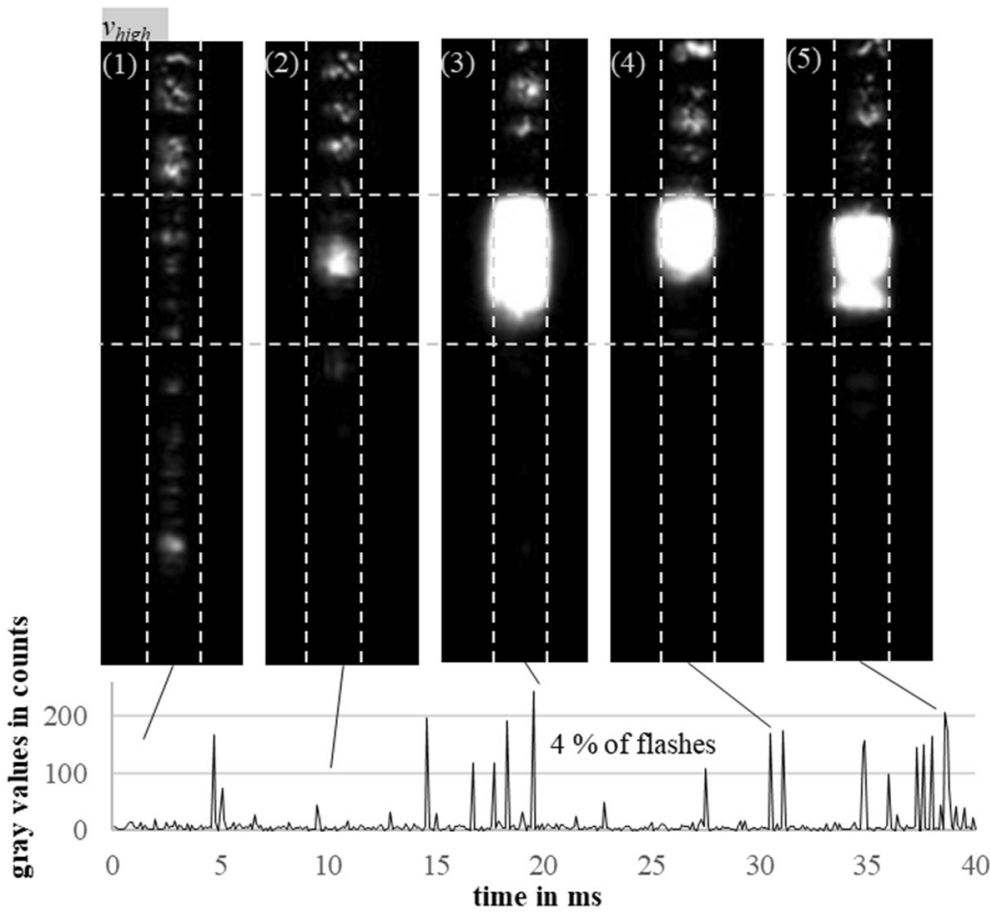

Fig. 6 Sequences of single frames taken from high-speed videos showing a higher intense of flashes at the cut front at the cutting velocity $v_{\text {high }}$. The vertical dashed lines indicate the edges of the cut kerf. The horizontal dashed lines show the upper and lower limits of the ROI. The diagrams below the images show the spatial average of the gray values of all pixels in the ROI of each frame plotted over time

cutting-edge surfaces at each velocity are shown in Fig. 7-b), 7-d) and 7-f). The comparison shows that flashes only occur when interrupted striations are formed.

Since it is unlikely that these flashes, which were recorded in the narrow spectral range between $500 \mathrm{~nm}$ and $550 \mathrm{~nm}$, stem from black-body radiation of the surface of the cut front they have to be caused by vaporization phenomena, as discussed in the following two sections.

\section{Temperature on the Surface of the Cut Front}

In order to determine whether the local flashes on the cut front are accompanied by vaporization, the process emissions in the ROI were analyzed with a spectrometer.

The spectral radiance $L_{p r}\left(\lambda, T_{p r}\right)$ recorded in the ROI at a cutting velocity $v_{\text {low }}$ is shown in Fig. 8. Discrete emission lines with low amplitude are visible in the spectral ranges between $380 \mathrm{~nm}$ and $450 \mathrm{~nm}$ and between $480 \mathrm{~nm}$ and $550 \mathrm{~nm}$. The process temperature was determined by a least-square fit of the gray-body spectrum to the measured spectrum in the ranges between $450 \mathrm{~nm}$ and $480 \mathrm{~nm}$ and between $580 \mathrm{~nm}$ and $730 \mathrm{~nm}$ - where no line emission was present. This yielded the temperature $T_{\text {prvlow }}=3080 \mathrm{~K}$ and $\varepsilon_{p y \text { vlow }}=0.32$. The temperature is below but close to the vaporization temperature of iron $T_{v, \text { iron }}=3134 \mathrm{~K}$ [18]. Hence, if at all vaporization takes place - as suggested by the just detectable emission lines in the recorded spectrum -, it can be assumed to be comparably infrequent and weak. 


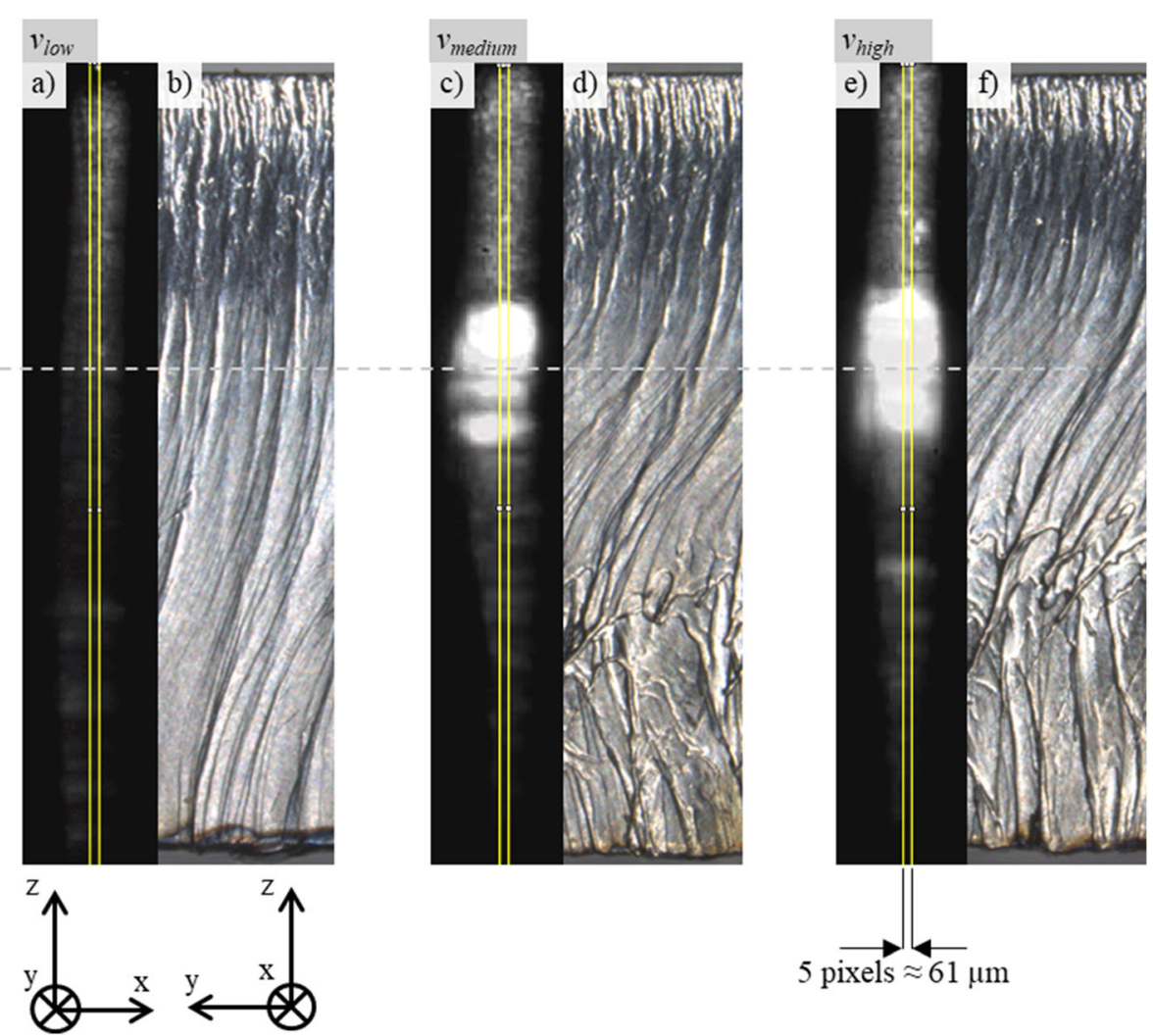

Fig. 7 "Stacked" images of process emissions on the cut fronts, extracted from high-speed video sequences of 100 frames of a process with a cutting velocity of $v_{\text {low }}$ (a), $v_{\text {medium }}$ (c) and $v_{\text {high }}$ (e). The corresponding surface of the cutting edge with regular striations and the cutting edges with interrupted striations are shown in (b), (d) and (f), respectively

The spectra (experimental and gray body) of the process with a cutting velocity of $v_{\text {medium }}$ are shown in Fig. 9. The more distinct emission lines indicate a stronger vaporization than at $v_{\text {low }}$. The least-square fit of the gray body spectrum to the experimental data yielded $\varepsilon_{\mathrm{prv} \text { vedium }}=0.24$ and $T_{p \text { pvmedium }}=3210 \mathrm{~K}$, which now exceeds the evaporation temperature of iron.

The same evaluation at the cutting velocity $v_{\text {high }}$ is shown in Fig. 10. Here the least square fit of the gray-body spectrum was applied only in the spectral range between $580 \mathrm{~nm}$ and $730 \mathrm{~nm}$, where the intensity of emission lines was small. The least-square fit of the gray body spectrum to the experimental data yielded $\varepsilon_{\mathrm{pv} v \text { high }}=0.27$. While the extracted process temperature $T_{p p v \text { vigh }}=3240 \mathrm{~K}$ was only slightly higher than $T_{p \text { v vmedium }}$ (but again exceeding the evaporation temperature), the intensity of the emission lines was significantly higher.

It should be noted, however, that although the temperature determined at $v_{\text {low }}$ is below and the ones determined at $v_{\text {medium }}$ and $v_{\text {high }}$ are higher than the evaporation temperature of iron, the differences are of the same order as the overall uncertainty of the measurements. Considering the uncertainty of $\pm 15 \mathrm{~K}$ of $T_{t s}$ mentioned above, the uncertainty of the process temperature $T_{p r, v h i g h}$ is $\pm 20 \mathrm{~K}$. Another uncertainty of $\pm 0.3 \mathrm{~K}$ of $T_{p r, v h i g h}$ comes from the uncertainty of the emissivity $\varepsilon_{T}$ of \pm 0.0082 [16]. The resulting uncertainty of the determined process temperature was therefore estimated 


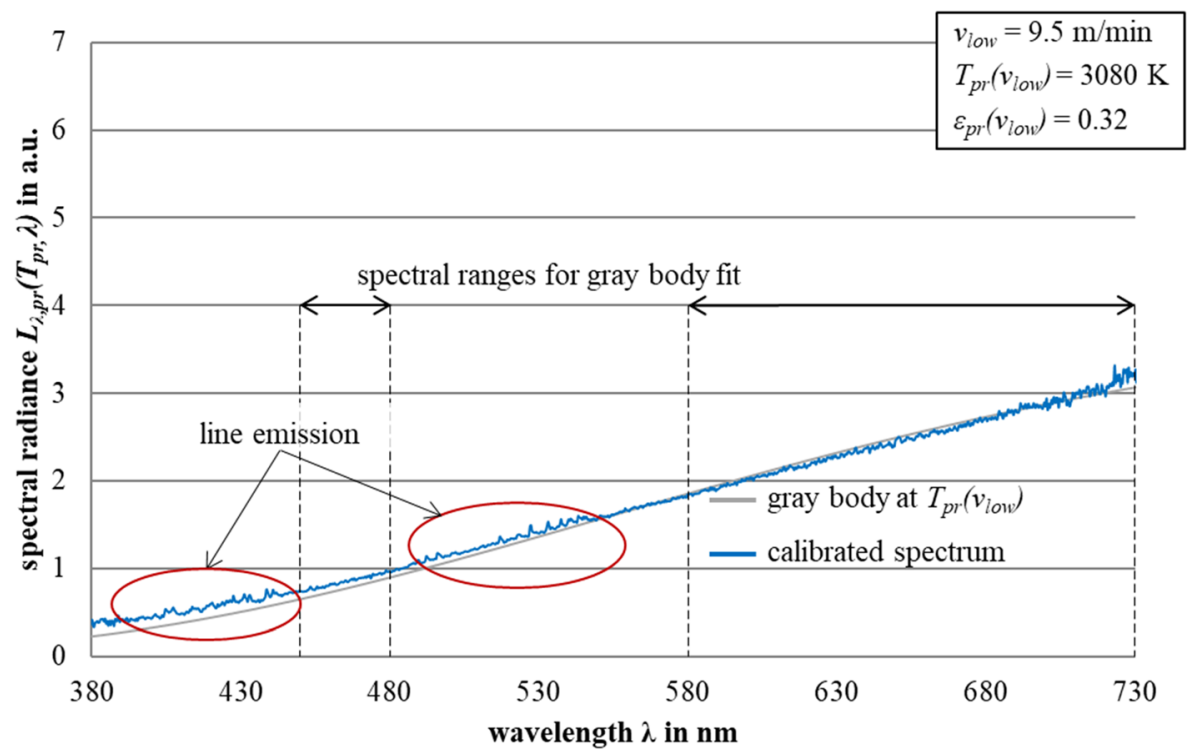

Fig. 8 Calibrated measured spectrum $L_{p r}\left(\lambda, T_{p r}\right.$ ) (blue line) of the cutting process, recorded at the cutting velocity $v_{\text {low }}$ (process with regular striations) and fitted spectrum of a gray body (gray line) with the temperature $T_{p r, \text { vlow }}=3080 \mathrm{~K}$ and the emissivity $\varepsilon_{p r}\left(v_{\text {low }}\right)=0.32$. The spectrum was recorded at the spectral ranges between $380 \mathrm{~nm}$ and $730 \mathrm{~nm}$, whereas the gray body fit was applied in a first range from $450 \mathrm{~nm}$ to $480 \mathrm{~nm}$ and a second range from $580 \mathrm{~nm}$ to $730 \mathrm{~nm}$ where no line emission was present

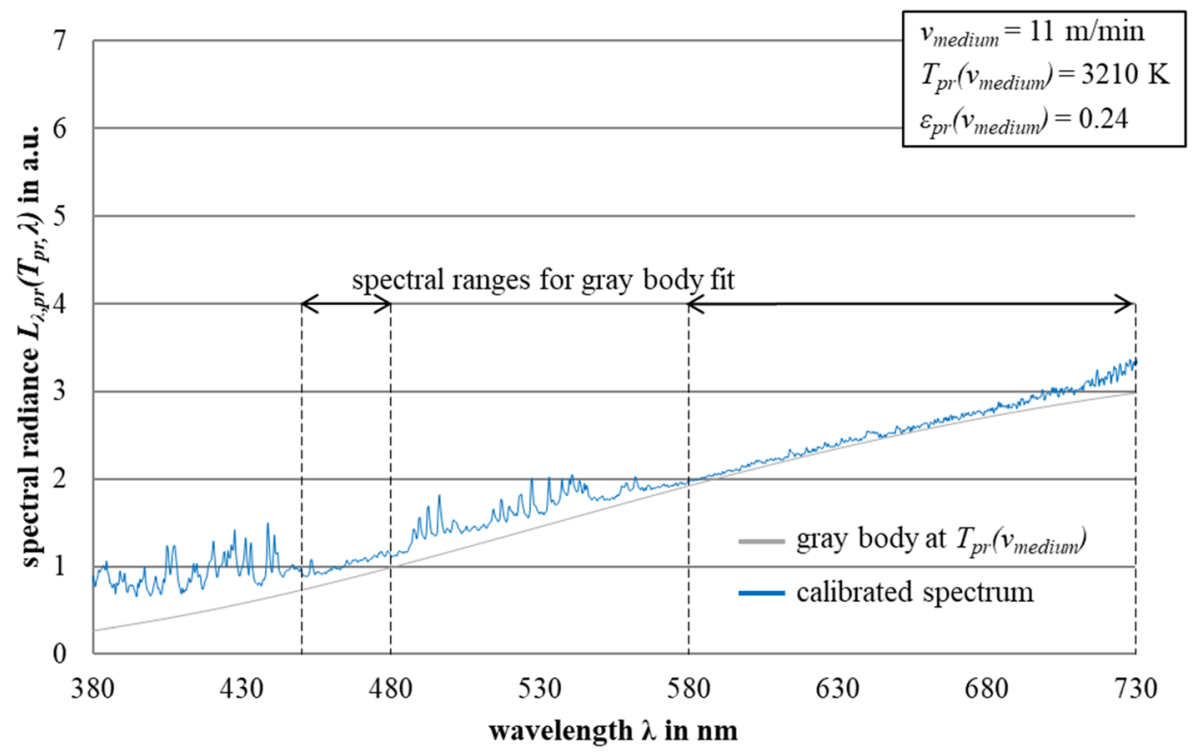

Fig. 9 Calibrated measured spectrum $L_{p r}\left(\lambda, T_{p r}\right.$ ) (blue line) of the cutting process, recorded at the cutting velocity $v_{\text {medium }}$ (process with interrupted striations) and fitted spectrum of a gray body (gray line) with the temperature $T_{\text {privmedium }}=3210 \mathrm{~K}$ and the emissivity $\varepsilon_{p r}\left(v_{\text {low }}\right)=0.24$. The spectrum was recorded at the spectral range between $380 \mathrm{~nm}$ and $730 \mathrm{~nm}$, whereas the gray body fit was applied in a first range from $450 \mathrm{~nm}$ to $480 \mathrm{~nm}$ and a second range from $580 \mathrm{~nm}$ to $730 \mathrm{~nm}$ where no line emission was present 


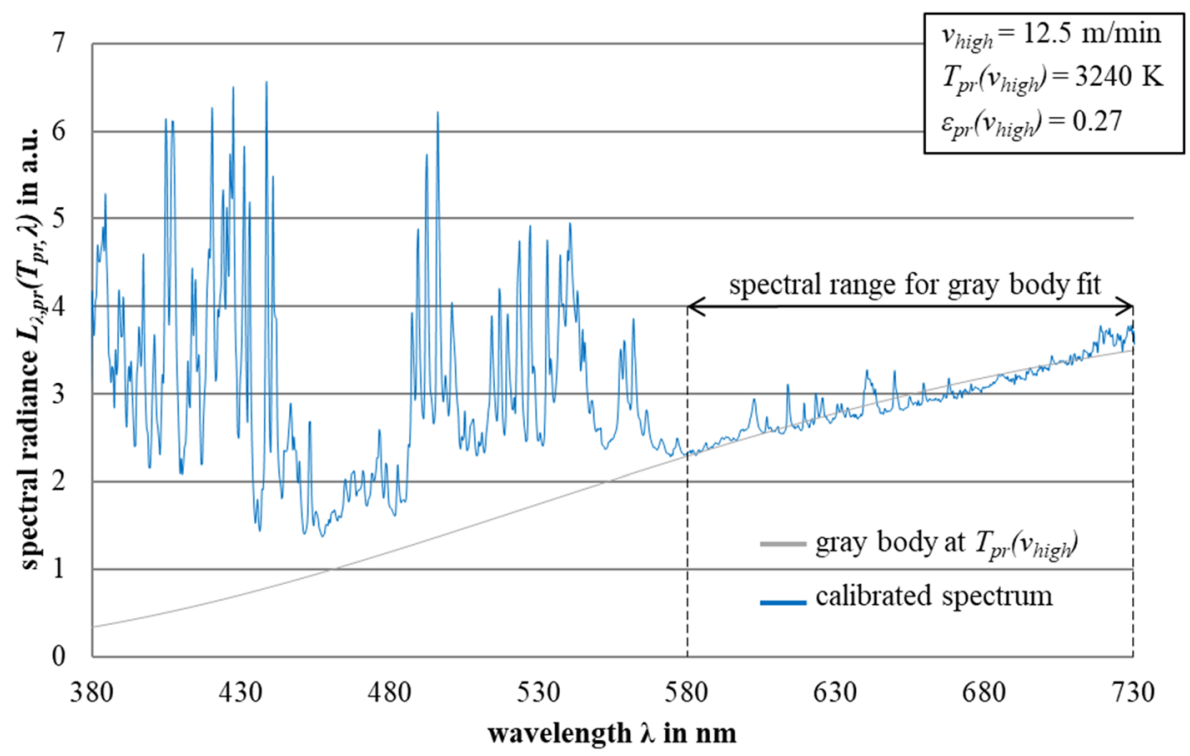

Fig. 10 Calibrated measured spectrum $L_{p r}\left(\lambda, T_{p r}\right)$ (blue line) of the cutting process, recorded at the cutting velocity $v_{\text {high }}$ (process with interrupted striations) and fitted spectrum of a gray body (gray line) with the temperature $T_{p r, v h i g h}=3240 \mathrm{~K}$ and the emissivity $\varepsilon_{p r}\left(v_{\text {low }}\right)=0.27$. The spectrum was recorded at the spectral range between $380 \mathrm{~nm}$ and $730 \mathrm{~nm}$, whereas the gray body fit was applied only in the spectral range from $580 \mathrm{~nm}$ to $730 \mathrm{~nm}$ where low line emission was present

to be $\pm 20.3 \mathrm{~K}$. Additionally, the evaluated process spectra were averaged over ten measurements. The absolute deviation of the temperature estimation within the ten measurements is $\Delta T_{\text {dev vhigh }}= \pm 292 \mathrm{~K}$. As a further confirmation that evaporation is indeed taking place at the elevated cutting speeds it is therefore shown in the following that the emission lines stem from the vaporized material of the processed sheet.

\section{Metal Vapor and its Temperature}

The spectral lines emitted by the process that were recorded at the cutting velocity $v_{\text {high }}$ were analyzed to determine whether they can be attributed to the evaporated base material, which consists for the most part of iron $(\mathrm{Fe})$.

Assuming a local thermodynamic equilibrium (LTE) [17], the emission coefficient $\varepsilon_{L}$ of a spectral line is given by

$$
\varepsilon_{L}=\frac{h \cdot c}{4 \pi} \cdot \frac{g_{21} \cdot A_{21}}{\lambda_{21}} \cdot \frac{n\left(T_{v}\right)}{Z\left(T_{v}\right)} \cdot \exp \left(-\frac{E_{21}}{k \cdot T_{v}}\right),
$$

where $g_{21}$ is the statistical weight, $A_{21}$ the Einstein's coefficient, $\lambda_{21}$ the emission wavelength and $E_{21}=\mathrm{E}_{2}-\mathrm{E}_{1}$ the energy difference between the higher and the lower level of the considered transition of the iron atom. The wavelength of the spectral lines are given by the NIST Atomic Spectra Database [19], which has been searched for the lines of $\mathrm{Fe} I$ in the spectral range between $380 \mathrm{~nm}-730 \mathrm{~nm}$. The index 1 describes the lower and the index 2 the higher energy level, whereas the index 21 describes the transition from the higher to the lower energy level of the atom. The electron density 
$n\left(T_{v}\right)$ and the partition function $Z\left(T_{v}\right)$ are assumed to be constant over the wavelength at the same degree of ionization and at the same temperature of the vapor $T_{v}$ in an LTE.

The spectral radiance

$$
L_{L}(\lambda)=L_{\lambda, p r}(\lambda)-\varepsilon_{p r} \cdot L_{\lambda, b l}\left(T_{p r}, \lambda\right)-L_{o f f s e t}
$$

of the line emission is derived from the measurements by subtracting the radiance of the gray body $\varepsilon_{p r} * L_{\lambda, b l}\left(T_{p r}, \lambda\right)$ and a constant offset $L_{o f f s e t}$ of $10^{5} \mathrm{~W} /\left(\mathrm{m}^{2} * \mu \mathrm{m} * \mathrm{sr}\right)$ from the calibrated measured process spectrum $L_{\lambda, p r}(\lambda)$. The measurements were found to exhibit a constant offset of $L_{\text {offset }}$ in the wavelength range between $495 \mathrm{~nm}$ and $533 \mathrm{~nm}$. The emission lines were examined in the wavelength range between $480 \mathrm{~nm}$ and $550 \mathrm{~nm}$ because the intensity of the emission lines is comparably strong in this spectral range and single lines can be distinguished more easily (see Fig. 11-a)).

Since the vapor can be assumed to have a high temperature $T_{v}$, the measured and theoretical spectral distribution and the relative values of the emitted lines $L_{L}$ were compared for temperatures $T_{v}$ ranging between $3000 \mathrm{~K}$ and $10,000 \mathrm{~K}$. A good match of the theoretical $\varepsilon_{L}$ with the measured $L_{L}$ was found for $T_{v}=7000 \mathrm{~K}$. Fig. 11-b) compares the measured spectrum $L_{L}(\lambda)$ with the calculated emission coefficient $\varepsilon_{L}$ for stimulated vapor of iron $(\mathrm{Fe} I)$ at a temperature $T_{v}=7000 \mathrm{~K}$.

The maximum difference of the wavelength of all peaks of the emission coefficients of $\mathrm{Fe} I$ from the NIST data base [19] and the peaks of the measured and examined emission lines were smaller than $0.11 \mathrm{~nm}$. This difference is smaller than the resolution of the measuring instrument of $0.3 \mathrm{~nm} /$ pixel, which shows that the values are in good agreement. The NIST data base [19] was also searched for Fe II lines, which could not be attributed to the examined spectrum. Hence the detected emission lines can reasonably well be attributed to the lines of $\mathrm{Fe} I$.

The above discussed flashes that can be observed during the cutting process, suggest that also the temperature experiences strong fluctuations. It should be noted that the spectrometer has a much longer exposure time than the camera, whereby the Planck fit yields an average temperature over time. Since the lines in the spectrum are associated to the short flashes, they can therefore be used to determine the higher vapor temperature present during these events.
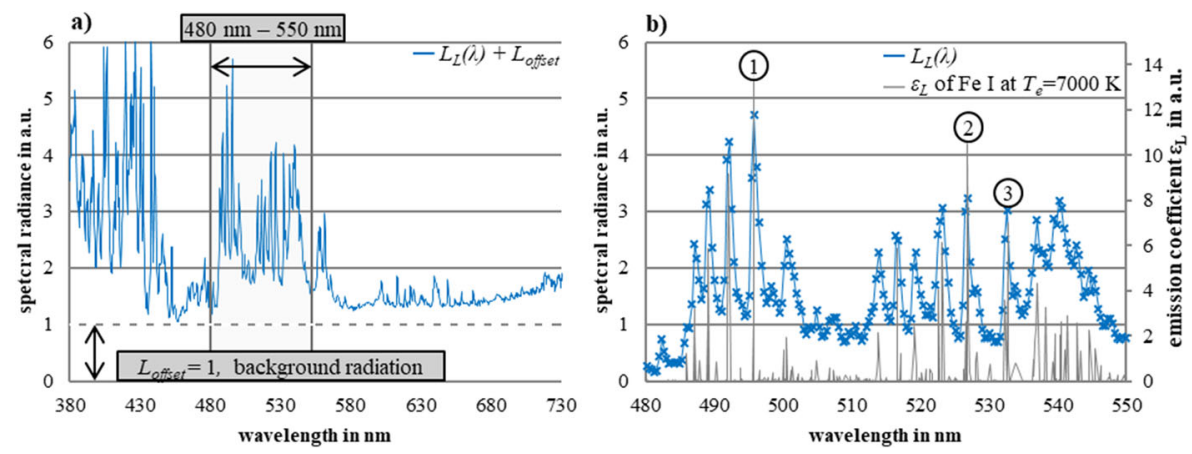

Fig. 11 Measured spectral radiance of the line emission $L_{L}(\lambda)+L_{\text {Offset }}$ from a cutting process with the cutting velocity $v_{\text {high }}$ where $L_{\text {Offset }}$ contains the background radiation (a). Detailed examination of the spectral radiance of the line emission $L_{L}(\lambda)$ in the spectral range between $480 \mathrm{~nm}$ and $550 \mathrm{~nm}$ where $L_{O f f s e t}$ was subtracted from the spectrum in comparison to the emission coefficient $\varepsilon_{L}(\lambda)$ as calculated for stimulated vapor of iron $(\mathrm{Fe} I)$ at a temperature of $T_{v}=7000 \mathrm{~K}$ (b); numbering of the examined emission lines, see Table 5 
In order to estimate this elevated temperature of the vapor $T_{v}$, the quotient

$$
\frac{\varepsilon_{L 1}\left(\lambda, T_{v}\right)^{!}}{\varepsilon_{L x}\left(\lambda, T_{v}\right)}=\frac{L_{L 1}(\lambda)}{L_{L x}(\lambda)}
$$

of the emission coefficients $\varepsilon_{L I}(\lambda)$ and $\varepsilon_{L x}(\lambda)$ is fit to the quotient of the intensity of the two closest lines $L_{L 1}(\lambda)$ and $L_{L x}(\lambda)$ of the measured spectrum. The indices indicate the number of the examined lines. The estimation was made by comparing line L1 (highest intensity) to line L2 and L3, see Table 5. As $n\left(T_{v}\right)$ and $Z\left(T_{v}\right)$ are assumed to be constant over the wavelength at the same degree of ionization and at the same temperature of the vapor $T_{v}$ in (6), both are cancelled from the fraction on the left of eq. (8). Using the eqs. (6) and (8), as well as the measured values for $L_{L}$ from Table 5, the temperature of the vapor $T_{v}$ can be estimated for each pair of emission lines. The quotient $L_{L 1} / L_{L 2}$ results in a vapor temperature of $7024 \mathrm{~K}$, while the quotient $L_{L 1} / L_{L 3}$ yields a vapor temperature of $6355 \mathrm{~K}$.

The investigation shows that interrupted striations only appear as soon as flashes occur, which are generated during the phase transition of the vaporization. Therefore, the critical temperature of the process emission which come along with the interrupted striations must be between the evaporation temperature of iron $\mathrm{T}_{\mathrm{v} \text {,iron }}$ and the estimated temperature $T_{v}$ of the vapor.

Although one can clearly state that these high temperatures are associated to violent evaporations during the flashes, it remains to clarify whether evaporation also occurs in between these flashes. Spectral measurements with a higher temporal resolution will be required to clarify this point.

\section{Discussion}

As mentioned before, the duration of the flashes and the associated local vaporization is much shorter than the exposure time of the sensor of the spectrometer. This leads to the assumption that the spectral radiance of the process

$$
L_{\lambda, p r}\left(\lambda, T_{p r}\right)^{!}=A \cdot \varepsilon_{p r} \cdot L_{\lambda, b l}\left(\lambda, T_{v, i r o n}\right)+(1-A) \cdot \varepsilon_{p r} \cdot L_{\lambda, b l}\left(\lambda, T_{v}\right)
$$

is composed of a contribution from the thermal emission of the surface with a temperature which is equal to the vaporization temperature of iron $\mathrm{T}_{\mathrm{v} \text {,iron }}$ and $\mathrm{a}$ contribution from the emission during the flashes in which the vapor reaches the

Table 5 Expected and measured wavelength of three distinctive emission lines L1, L2 and L3 and the measured intensity $L_{L}$. The theoretical values of the wavelength were calculated for a vapor temperature of $T_{v}=7000 \mathrm{~K}[19]$

\begin{tabular}{llll}
\hline Line-No. & \multicolumn{2}{l}{ Expected wavelength } & \multicolumn{2}{l}{ Measured values } \\
\cline { 3 - 4 } & $\lambda_{n m}[\mathrm{~nm}]$ & $\lambda_{m e}[\mathrm{~nm}]$ & $L_{L}$ [a.u.] \\
\hline L1 & 495.75961 & 495.86 & 4.71 \\
L2 & 526.95366 & 526.97 & 3.23 \\
L3 & 532.80381 & 532.73 & 3.03 \\
\hline
\end{tabular}




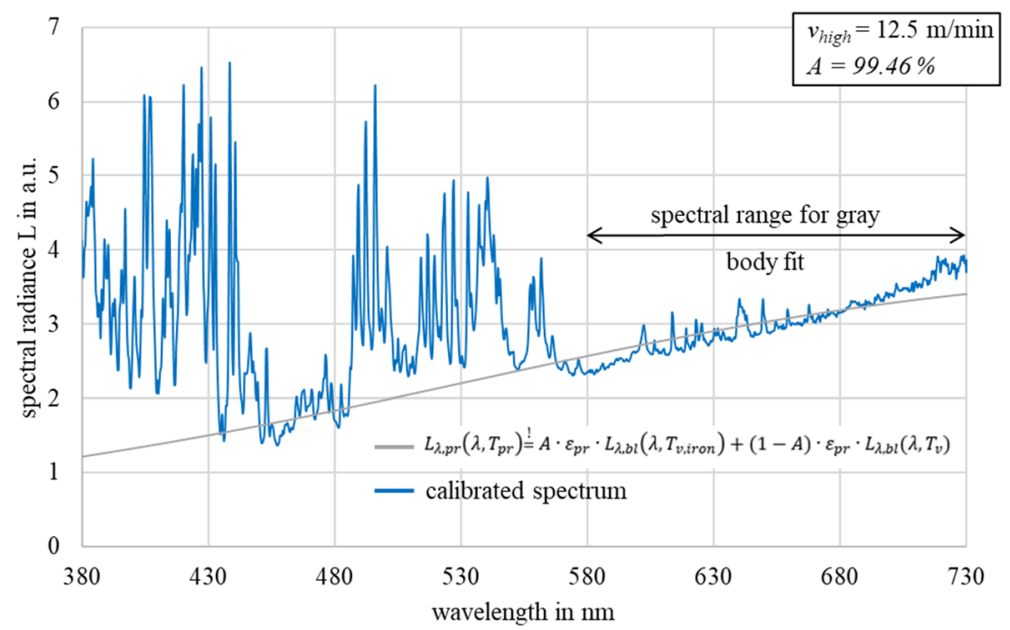

Fig. 12 Calibrated measured spectrum $\mathrm{L}_{\mathrm{pr}}\left(\lambda, \mathrm{T}_{\mathrm{pr}}\right.$ ) (blue line) of the cutting process, recorded at the cutting velocity $v_{\text {high }}$ (process with interrupted striations) and fitted spectrum of a gray body (gray line) with the temperature composed of two components: a contribution from the surface at vaporization temperature of iron $\mathrm{T}_{\mathrm{v}, \text { iron }}$ weighted by the temporal faction $\mathrm{A}=99.46 \%$ of the process without flashes and a contribution from the emission of the vapor at the elevated temperature $\mathrm{T}_{\mathrm{v}}$ weighted by 1-A. The gray body fit was applied only in the spectral range from $580 \mathrm{~nm}$ to $730 \mathrm{~nm}$ where the line emission is weak

elevated the temperature of $\mathrm{T}_{\mathrm{v}}$. The two contributions are weighted by the fractions of time A and 1-A without and with flashes, respectively.

The recorded spectral radiance $\mathrm{L}_{\mathrm{pr}}\left(\lambda, \mathrm{T}_{\mathrm{pr}}\right)$ at a cutting velocity $\mathrm{v}_{\text {high }}$ is shown in Fig. 12 . The temporal component A was determined by a least-square fit of the gray-body spectrum, which is described in eq. (9), to the measured spectrum in the range between $580 \mathrm{~nm}$ and $730 \mathrm{~nm}$. This yielded the temporal component $\mathrm{A}=99.46 \%$. This means that the temperature on the cut front in the ROI is $\mathrm{T}_{\mathrm{v}}=7000$ for only $0.54 \%$ of the duration of the measurement, while the rest of the time the temperature is $T_{v, \text { iron }}=3134 \mathrm{~K}$. This result is consistent with the observation in the high-speed videos at $\mathrm{v}_{\text {high }}$, where only $4 \%$ of all captured images show flashes on the surface of the cutting front, see Fig. 6.

This approach assumes that there are only these two temperatures present. Based on the low temporal resolution of the spectrometer, however, it cannot be clearly determined which temperatures actually prevail between the flashes on the cut front. To clarify whether vaporization on the cut front exists during the entire cutting process or only during the flashes, measurements with a higher resolution would be necessary in the future. The presented measurements however show, that the evaporation is very strong, and temperatures are very high during the flashes. The results show that vaporization increases at higher cutting speeds. On the other hand, the vaporization and generation of interrupted striations can be controlled and reduced by reducing the cutting speed.

\section{Conclusion}

Both, the high-speed videos and the spectrometric measurements indicate that the transition to an interrupted striation pattern is associated to a greater degree with flashes caused by local vaporization with high vapor temperatures, than with increasing temperatures on the 
surface of the cut front. It seems likely that most of the material evaporation takes place in the upper part of the cut front, which does not mean that there is no vaporization in other parts of the cut front as well. The vaporization might disturb the melt flow and cause the formation of interrupted striations, as stated in [10]. Further investigations will be required to verify this finding, but in any case, the observation of the flashes can be used to foresee cutting flaws and to identify the generation of interrupted striations.

Funding Information Open Access funding provided by Projekt DEAL.

Open Access This article is licensed under a Creative Commons Attribution 4.0 International License, which permits use, sharing, adaptation, distribution and reproduction in any medium or format, as long as you give appropriate credit to the original author(s) and the source, provide a link to the Creative Commons licence, and indicate if changes were made. The images or other third party material in this article are included in the article's Creative Commons licence, unless indicated otherwise in a credit line to the material. If material is not included in the article's Creative Commons licence and your intended use is not permitted by statutory regulation or exceeds the permitted use, you will need to obtain permission directly from the copyright holder. To view a copy of this licence, visit http://creativecommons.org/licenses/by/4.0/.

\section{References}

1. Wandera, C., Salminen, A., Kujanpaa, V.: Inert gas cutting of thick-section stainless steel and mediumsection aluminum using a high power fiber laser. Journal of Laser Applications. 21(3), 154-161 (2009)

2. Bocksrocker, O., Berger, P., Fetzer, F., Rominger, V., Graf, T.: Influence of the real geometry of the laser cut front on the absorbed intensity and the gas flow. Lasers Manuf Mater Process. 24, 52006 (2018)

3. Olsen, F.O.: Laser Cutting from CO2 Laser to Disc or Fiber Laser - Possibilities and Challenges, Proceedings of the Laser Materials Processing Conference (ICALEO) (Paper 101) (2011)

4. Olsen, F.O.: An evaluation of the cutting potential of different types of high power lasers. Proceedings of the Laser Materials Processing Conference (ICALEO). (2006)

5. Olsen, F.O.: Fundamental mechanisms of cutting front formation in laser cutting. In: Beyer, E., Cantello, M., La Rocca, A.V., Laude, L.D., Olsen, F.O., Sepold, G. (eds.) SPIE, p. 402 (1994)

6. Petring, D., Schneider, F., Wolf, N.: Some answers to frequently asked questions and open issues of laser beam cutting. Proceedings of the Laser Materials Processing Conference (ICALEO). (2012)

7. Petring, D., Molitor, T., Schneider, F., Wolf, N.: Diagnostics. Modeling and Simulation: Three Keys Towards Mastering the Cutting Process with Fiber, Disk and Diode Lasers, Physics Procedia. 39, 186-196 (2012)

8. Onuseit, V., Ahmed, M.A., Weber, R., Graf, T.: Space-resolved spectrometric measurements of the cutting front. Phys Procedia. 12, 584-590 (2011)

9. V. Onuseit, M. Jarwitz, R. Weber, T. Graf, Influence of cut front temperature profile on cutting process, Proceedings of the Laser Materials Processing Conference (ICALEO) (Paper 104) (2011) 34-39

10. Sparkes, M., Gross, M., Celotto, S., Zhang, T., O’Neill, W.: Practical and theoretical investigations into inert gas cutting of 304 stainless steel using a high brightness fiber laser. J Laser Appl. 20(1), 59-67 (2008)

11. Sichani, E.F., Kohl, S., Duflou, J.R.: Plasma detection and control requirements for $\mathrm{CO} 2$ laser cutting. CIRP Ann Manuf Technol. 62(1), 215-218 (2013)

12. Kaplan, A.F.H.: Local flashing events at the keyhole front in laser welding. Opt Lasers Eng. 68, 35-41 (2015)

13. Kaplan, A.F.H., Matti, R.S.: Absorption peaks depending on topology of the keyhole front and wavelength. Proceedings of the Laser Materials Processing Conference (ICALEO). (2014)

14. O. Bocksrocker, T. Hesse, S. Epple, Deutsche Patentanmeldung (engl. german patent application) Anmeldenummer (registration number) 102016208264 (2016)

15. OSRAM: Display / Optic, Technical Information: WI 17/G Tungsten Strip Lamp, Berlin (2008)

16. de Vos, J.C.: A new determination of the emissivity of tungsten ribbon. Physica. 20, 690-714 (1954)

17. Bernhard, F.: Handbuch der Technischen Temperaturmessung, second. Auflage edn. Springer Vieweg, Heidelberg (2014)

18. Information on http://www.periodictableontheweb.com/periodensystem/element-Iron.php

19. Information on http://physics.nist.gov/PhysRefData/ASD/lines_form.html

Publisher's Note Springer Nature remains neutral with regard to jurisdictional claims in published maps and institutional affiliations. 